\title{
Epidermal curettage technique (ECT) for tissue harvest from the donor area for melanocyte autologous grafting in cases of vitiligo
}

\author{
Carlos D`Apparecida Santos Machado Filho ${ }^{1}$
}

Fabio Roismann Timoner ${ }^{1}$

DOI: http://dx.doi.org/10.1590/abd1806-4841.20142865

\begin{abstract}
Vitiligo is a dermatosis requiring complex treatment. In clinically stable cases, melanocyte autologous grafting has shown good results, using different methodologies for obtaining the donor area: dermatomes, punches, blisters. However, these techniques are complex and require specific instruments. This study presents a simple technique for melanocyte harvest that has been performed in our service for more than ten years and includes epidermal curettage of the donor area, grafting of the obtained material, which was homogenized with saline or hyaluronic acid, on the achromic area, and the application of a semipermeable dressing that is removed 1 week later.
\end{abstract}

Keywords: Curettage; Melanocytes; Skin transplantation; Surgical procedures, minor; Vitiligo

Vitiligo is a dermatosis with a prevalence of $1 \%$ in the world population and has a great negative impact on patient quality of life. ${ }^{1}$ Its causes are multifactorial, and its treatment depends both on the extent of the affected body area and on the degree of disease activity. ${ }^{1}$

In cases of extensive vitiligo, either active or stable, the treatment consists of combining several therapeutic modalities, such as light therapy - especially narrowband ultraviolet B (NB-UVB) -, systemic corticotherapy - in cases of active vitiligo -, topical corticotherapy, topical corticotherapy, vitamin D analogues, and topical immunomodulators such as calcineurin inhibitors. ${ }^{2}$

Melanocyte grafting techniques have proven to be effective in stable cases and are also indicated simultaneously with the above mentioned treatments. ${ }^{2-5}$

Melanocyte grafting may be performed with the culture of these cells, which forms a fluid suspension after being fragmented by trypsin. This method allows treating more extensive lesions using a smaller donor area, due to the increase in cellularity and the formation of a fluid vehicle. ${ }^{2,46}$

Grafting may also be performed with non-cultured cells. ${ }^{6,7}$ The methodology for punch minigrafting is already well established, but it may promote a peculiar appearance that is not very esthetically pleasing for the patient, because there is the formation of progressive repigmentation halos adjacent to grafts, and grafts may remain with an elevated appearance for an indeterminate period. ${ }^{2}$
The most reported techniques for epidermis harvest without punching are performed using either automatic dermatomes or Blair's knives, or involve the formation of suction bubbles combined or not with the application of infrared light. ${ }^{2}$ The harvested material is fully inserted as a partial graft to the lesion previously abraded or de-epidermized by laser or ultrasound. ${ }^{3,5}$

This study presents the epidermal curettage technique (ECT), which has not been described yet in the literature as a harvest method for melanocyte grafting along with already routinely used techniques. This technique has been performed at the Dermatology Service of Faculdade de Medicina do ABC since 2000, initially in studies that aimed to analyze the impact of grafting with harvest by epidermal scraping and study melanocyte activation by reverse transcription polymerase chain reaction for tyrosinase messenger RNA in achromic areas and subsequently in the treatment of stable vitiligo in small areas (Figures 1A-1B), as demonstrated by Machado Filho et al., who found clinical and laboratory signs of repigmentation in all the 40 patients who received grafts using the ECT. ${ }^{8}$ There was a significant increase in the percentage of melanocytes in grafted areas, in comparison with control areas (that were only curetted) ${ }^{8}$ Evidence of a discrete increase in the number of melanocytes in the control area was observed, a fact that was confirmed in other studies conducted by Barros et al. and Quezada et al., ${ }^{9,10}$

\footnotetext{
Received on 12.06.2013

Approved by the Advisory Board and accepted for publication on 15.08.2013

* Work conducted at the Dermatology Course at Faculdade de Medicina do ABC - Santo André (SP), Brazil.

Conflict of Interests: None.

Faculdade de Medicina do ABC (FMABC) - Santo André (SP), Brazil.

(C)2014 by Anais Brasileiros de Dermatologia
} 
The ECT is performed with a completely pigmented and stable donor area, usually the sacral region (Figure 1A). The size of donor and recipient areas should have a ratio of 1:4. After these areas are demarcated and undergo antisepsis, anesthesia is obtained with $2 \%$ lidocaine and vasoconstrictor, and a curettage of the entire region is performed with a sterile curette until the Auspitz's sign becomes visible (Figure $1 \mathrm{~B}$ and $1 \mathrm{C}$ ). The obtained material is placed into a sterile tube and mixed with saline or hyaluronic acid until achieving a pasty consistency and forming a "paste of melanocytes" (Figure 1D). A des-epidermialization is performed in the recipient area using the same technique, but the obtained skin is discarded. Finally, the "paste of melanocytes" is applied over the recipient area, which is occluded with a semipermeable membrane dressing that is removed 1 week later (Figure $1 \mathrm{E}$ and $1 \mathrm{~F}$ ).

Adhesive semipermeable dressings may detach grafted cells from their beds if the patient moves the region, which is why non-adhesive and perforated dressings have been preferred, since they make it possible to remove transudate and thus reduce the degree of humidity that may also lead to graft detachment.

Mixing hyaluronic acid with the obtained grafts is a more recent technique that may increase melanocyte viability or even form a vehicle with better adherence and fixation to the recipient areas. ${ }^{4.6}$
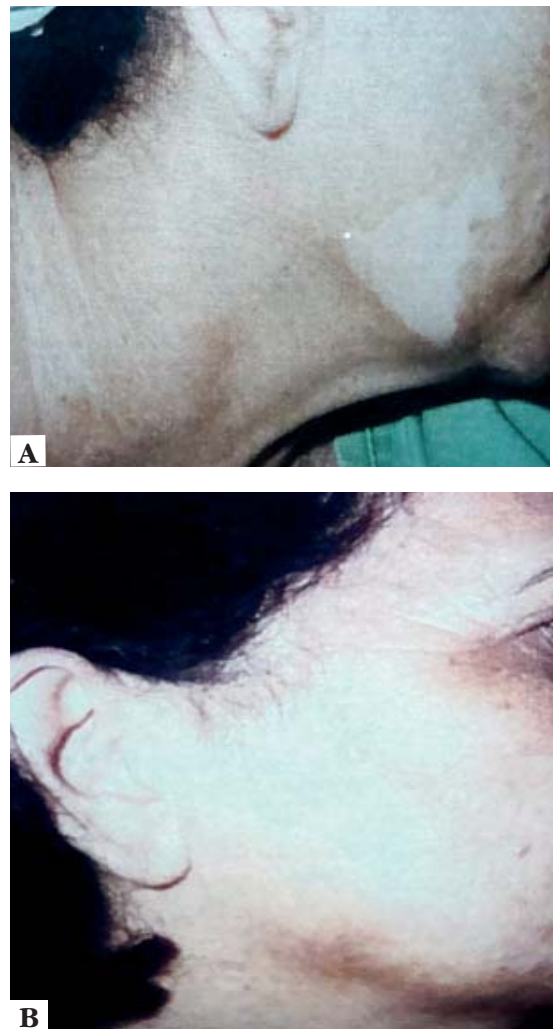

Figure 2: Case of ECT. A.Pretreatme nt; B. Posttreatment
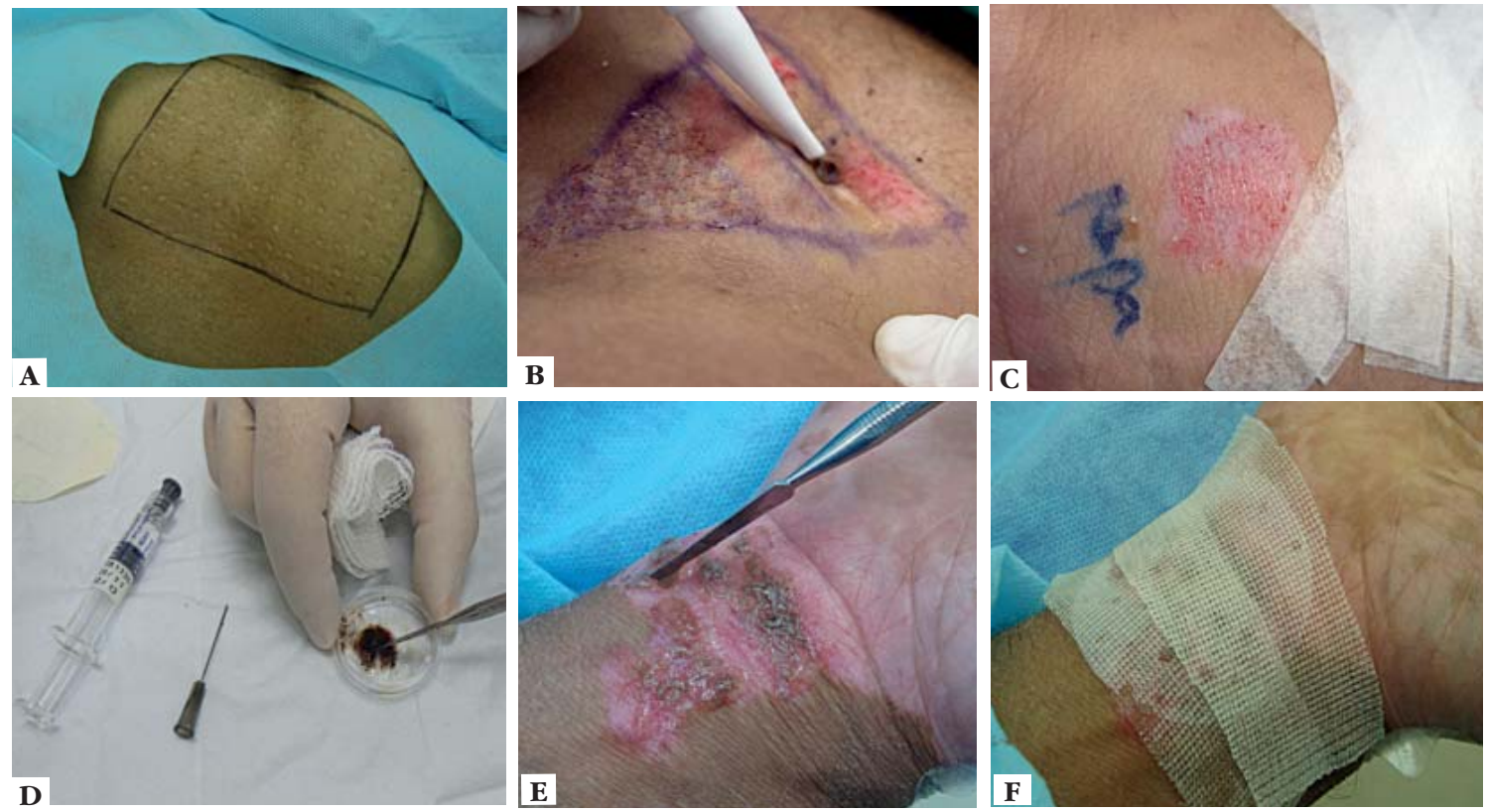

Figure 1: Epidermal curettage technique (ECT). A. Donor area; B. Epidermal curettage; C. Auspitz's sign; D. Preparation of the paste of melanocytes; E. Grafting on the recipient area; F. Dressing 
The ECT has the advantage of being simpler than the methods mentioned before, having a low cost, and being able to be performed outside the hospital setting using only basic instruments.

This harvest technique has already been included in our setting together with the other harvest techniques reported in this study, whether followed by cell culture or non-cultivated melanocyte suspension. Due to its simplicity, the ECT may make it easier to obtain melanocytes, thus optimizing current techniques of cell processing in lesions of stable vitiligo, mainly in segmental types. In terms of healing of the donor area, the ECT has the advantage of promoting diffuse, more homogeneous and faster repigmentation when compared with the punch grafting technique, and allowing for the donor area to be reused (Figure 2).

Additional studies should be conducted with the purpose of obtaining more data on cell viability and on the possibility of promoting a greater dilution of the sample leading to a consequent expansion with the ECT and maybe comparing this technique with usually performed methods.

\section{REFERENCES}

1. Ali A, Lesley MF, Meaghan D, Vesna PR. Vitiligo: a comprehensive overview Part I. Introduction, epidemiology, quality of life, diagnosis, differential diagnosis, associations, histopathology, etiology, and work-up. J Am Acad Dermatol 2011;65:473-91.

2. Felsten LM, Alikhan A, Petronic-Rosic V. Vitiligo: A compreensive overview: Part II: Treatment options and approach to treatment. J Am Acad Dermatol. 2011;65:493-514.

3. Machado Filho CDAS. Implantes em Vitiligo. [dissertação]. São Paulo (SP): Universidade Federal de São Paulo; 1986. 110 p.

4. Goh BK, Chua XM, Chong KL, de Mil M, van Geel NA. Simplified cellular grafting for treatment of vitiligo and piebaldism: the "6-well plate" technique. Dermatol Surg. 2010;36:203-7.

5. Tsukamoto K, Osada A, Kitamura R, Ohkouchi M, Shimada S, Takayama 0. Approches to repigmentation of vitiligo skin:new treatment with ultrasonic abrasion,seed grafting and psoralen plus ultraviolet A therapy. Pigment Cell Res. 2002;15:331-4.

6. Mulekar SV, Al Issa A, Al Eisa A. Treatment of Vitiligo on Difficult Sites Using Autologous Noncultured Cellular Grafting. Dermatol Surg. 2009;35:66-71.

7. Gauthier Y, Surleve-Bazeille JE. Autologus graftings with non cultured melanocytes: a simplified method for treatment of depigmented lesions. J Am Acad Dermatol. 1992;26:191-4.

8. Machado Filho CD, Almeida FA, Proto RS, Landman G. Vitiligo: analysis of grafting versus curettage alone, using melanocyte morphology and reverse transcriptase polymerase chain reaction for tyrosinase mRNA. Sao Paulo Med J. 2005;123:187-91.

9. Barros JA, Machado Filho CD AS, Martins CM, Pettinati J, Pinhal MAS. Vitiligo: histological and clinical evaluation after sequential curettage. An Bras Dermatol. 2007;82:327-35

10. Quezada N, Machado Filho CA, De La Sotta P, Uribe P. Melanocytes and keratinocytes transfer using sandpaper technique combined with dermabrasion for stable vitiligo. Dermatol Surg. 2011;37:192-8.

\section{MAILING ADDRESS:}

Fabio Roismann Timoner

Av. Príncipe de Gales, 821

Príncipe de Gales

09060-650 - Santo André - SP

Brazil

E-mail:drfabiotimoner@hotmail.com

How to cite this article: Machado Filho CDAS, Timoner FR. Epidermal curettage technique (ECT) for tissue harvest from the donor area for melanocyte autologous grafting in cases of vitiligo. An Bras Dermatol. 2014;89(4):681-3. 